\title{
Intrants biologiques et politiques agricoles en Amérique du Sud : entre ruptures et continuités
}

\author{
Frédéric Goulet
}

En Amérique du Sud, les politiques publiques s'intēressent de près aux technologies alternatives aux intrants chimiques agricoles (pesticides et engrais]. Certains pays sud-américains soutiennent les intrants biologiques, dits aussi biointrants, avec des programmes nationaux incitatifs et la transformation des réglementations. L'Argentine, le Brésil et la Colombie occupent un rôle pilote. Cette promotion des biointrants n'est néanmoins pas pensée dans une optique de rupture avec les modèles industriels de production agricole, dont les États tirent une grande partie de leurs recettes fiscales grâce aux exportations. Ils cherchent plutôt à aménager une coexistence entre intrants chimiques et biologiques dans le cadre d'une transition tournée vers la bioéconomie. La promotion des biointrants rencontre en ce sens les attentes de nombreux agriculteurs sud-américains et celles de l'industrie des intrants agricoles, qui se diversifie depuis une dizaine d'années dans ces technologies. Cette dynamique industrielle vient en partie se heurter à des mouvements d'agriculteurs défendant la production à la ferme des intrants biologiques.

\section{Un tournant microbiologique et biotechnologique}

Pour réduire l'usage des pesticides, les pays agricoles élaborent des politiques publiques encourageant le biocontrôle. La France, par exemple, met en œuvre sa " stratégie nationale de déploiement du biocontrôle 20202025 ». L'Amérique du Sud, en particulier les grands pays comme le Brésil et l'Argentine, n'échappe pas à cette tendance, même si l'intention de réduire les pesticides reste relativement modérée par rapport à l'Europe.

Depuis le milieu des années 2010, plusieurs pays sudaméricains, avec notamment l'appui de l'Institut interaméricain de coopération pour l'agriculture (IICA), ont lancé des initiatives allant dans ce sens. Argentine, Brésil et Colombie sont précurseurs dans ces changements. Ils ont organisé leur action autour d'une catégorie d'intrants agricoles plus englobante que la définition du biocontrôle (voir encadré p. 2) : les biointrants (bioinsumos), qui incluent à la fois les produits de biocontrôle et les biofertilisants. Ces derniers sont incarnés par les bioinoculants, qui ont connu un boom considérable avec l'essor du soja depuis la fin des années 1990 et qui sont utilisés en traitement des semences pour réduire les apports d'engrais azotés. En Argentine et au Brésil, une industrie nationale florissante s'est développée autour de ces technologies à partir d'interactions entre de petites et moyennes entreprises et les universités publiques.

Ce tournant microbiologique et biotechnologique s'observe aussi dans le secteur du biocontrôle, pour lequel la recherche-développement se concentre aujourd'hui sur les bactéries et les champignons, reléguant souvent au second plan la macrofaune (insectes par exemple) utilisée contre des bioagresseurs des cultures. Les chiffres d'enregistrement de nouveaux produits sont de ce point de vue sans équivoque. Au Brésil, entre 2000 et 2020 , les produits de biocontrôle à base de microorganismes ont constitué en moyenne $60 \%$ des nouveaux biointrants enregistrés $[17,6 \%$ seulement pour ceux issus de macroorganismes, le reste étant des médiateurs chimiques et autres substances naturelles]. Mais cette proportion a augmenté drastiquement sur la période pour atteindre $80 \%$ en 2020. Également, plus de la moitié de ces produits enregistrés ces 20 années sont des bioinsecticides.

Les fronts d'innovation autour des biointrants sont ainsi avant tout biotechnologiques, comme en témoigne en Argentine leur prise en charge institutionnelle par la Commission nationale consultative sur les biotechnologies agricoles (CONABIA, Comisión Nacional Asesora de Biotecnología Agropecuaria]. 


\section{Du biocontrôle aux biointrants : définitions}

Le biocontrôle désigne un ensemble de techniques d'origine biologique utilisées en protection des plantes pour lutter contre leurs bioagresseurs (microorganismes, insectes, acariens, nématodes, etc.). On distingue généralement quatre principaux types d'agents de contrôle biologique

$>$ les macroorganismes auxiliaires invertébrés, comme les insectes et les acariens;

> les microorganismes (champignons, bactéries, virus] utilisés pour protéger les cultures contre les ravageurs et les maladies ou pour stimuler la vitalité des plantes;

$>$ les médiateurs chimiques, incluant surtout les phéromones d'insectes, qui permettent le contrôle des populations d'insectes en utilisant la méthode de confusion sexuelle ou en facilitant l'attraction des ravageurs dans des pièges

> les substances naturelles d'origine végétale, animale ou minérale.
Les biofertilisants sont des produits d'origine biologique utilisés pour accroître la fertilité des sols. Ce sont des produits à base de matières organiques (déjections animales, résidus végétaux, composts], ou des solutions à base de microorganismes, comme les bioinoculants.

Les bioinoculants sont des produits essentiellement à base de bactéries fixatrices d'azote sélectionnées et améliorées en laboratoire. Ces bactéries vivent naturellement en symbiose avec les racines des légumineuses [telles que le soja, le pois chiche, les haricots], capturant les éléments chimiques de l'air et du sol et les restituant sous une forme assimilable à la plante. Les bioinoculants sont utilisés sur les légumineuses et sur d'autres cultures, comme la canne à sucre.

Ces intrants biologiques destinés à l'agriculture sont nommés plus généralement biointrants [bioinsumos] en Amérique du Sud.

\section{Agrochimie et biointrants : rapprochements et continuités}

En Amérique du Sud comme sur d'autres continents, I'usage d'intrants d'origine biologique est une pratique ancestrale. Les agricultures traditionnelles et vivrières, ou plus récemment l'agriculture biologique tournée vers les marchés intérieurs ou l'exportation, ont recours à des préparations et des technologies diverses, que ce soit pour fertiliser les sols ou pour assurer la santé des plantes cultivées.

L'expansion d'une industrie des biointrants est plus récente et a pris un tournant spécifique ces dernières années. Elle s'inscrit tout d'abord dans l'optique du développement d'une bioéconomie qui, si elle est destinée à l'ensemble du secteur agricole, est résolument tournée vers l'utilisation des ressources biologiques dans des systèmes de production intensifs. La biodiversité est vue comme une richesse à explorer et à exploiter pour rendre plus durable sur le plan écologique le modèle agricole hérité de la Révolution verte. Le développement des biointrants s'inscrit ensuite dans la transformation de l'industrie des intrants agricoles à l'échelle sud-américaine et également mondiale.

Les entreprises multinationales agrochimiques ont investi dans le secteur des biointrants en opérant de nombreux rachats d'entreprises nationales. Elles s'implantent ainsi sur ce front technologique émergent, jouissant d'une croissance élevée sur les marchés sud-américains - au Brésil les ventes de produits de biocontrôle ont augmenté de 15 \% entre 2018 et 2019 (voir encadré p. 3). Ce faisant, elles poursuivent leur diversification opérée vers les biotechnologies depuis les investissements réalisés dans le domaine semencier, mais appliquée cette fois à la santé et à la nutrition des plantes. Ce mouvement est illustré par la trajectoire de l'Association brésilienne des industries du biocontrôle (ABC Bio, Associação Brasileira das Empresas de Controle Biológico). Regroupant à l'origine des petites et moyennes entreprises du sud du Brésil, elle a vu arriver à partir du milieu des années 2010 de grands groupes internationaux de l'agrochimie investissant dans le biocontrôle. En 2020, cette mutation a marqué un nouveau pas : ABC Bio a fusionné avec les alliances brésiliennes des industries de l'agrochimie et des biotechnologies pour former l'association CropLife Brasil.

Ces rapprochements industriels montrent que la substitution radicale des intrants chimiques par des intrants biologiques n'est pas l'objectif visé. Il s'agit plutôt d'une diversification industrielle et d'investissements dans un secteur prometteur. Les intrants biologiques attirent en effet de grandes exploitations agricoles et des filières de plus en plus concernées par les exigences sanitaires des pays importateurs. Les intrants biologiques sont des technologies alternatives aux intrants chimiques, par leur nature et leurs modes d'action, mais leur développement industriel les inscrit dans une forme de continuité, ou du moins de coexistence, avec le modèle chimique dominant. Les politiques publiques sud-américaines promeuvent d'ailleurs cette vision d'une transition très progressive, en mettant en avant les intrants biologiques comme une solution d'avenir, complémentaire à court et moyen termes des intrants chimiques.

\section{Politiques publiques : de l'adaptation règlementaire aux plans intégrateurs}

Les politiques sud-américaines en faveur des biointrants investissent différents domaines d'action. Le plus important est probablement celui de l'adaptation des cadres règlementaires d'évaluation et d'homologation des produits, jusqu'à présent construits pour les intrants chimiques. Cette action est primordiale, car c'est d'elle que dépend la mise sur le marché des intrants biologiques. Or ces derniers sont encore en majorité évalués selon les mêmes critères que les produits chimiques.

Cette adaptation des cadres règlementaires est toutefois difficile. Les services publics en charge de l'évaluation comptent peu de biologistes, les laboratoires habilités sont peu nombreux et la réforme des dispositions existantes se heurtent souvent à la bureaucratie. Cette adaptation 
est pourtant essentielle pour stimuler l'innovation et pour que l'État puisse jouer son rôle de garant de la qualité et de l'innocuité des produits mis sur le marché. La Colombie est le seul pays à disposer d'une législation spécifıque aux intrants biologiques, mais elle n'a pas élaboré de programme intégrateur pour la promotion des biointrants, comme l'ont fait récemment l'Argentine et le Brésil. Ces deux pays ont en effet mis en place, au travers de plans gérés par les ministères de l'agriculture, des instruments agissant sur plusieurs leviers pour dynamiser la production et l'usage des intrants biologiques. Ce sont par exemple des dispositifs pour favoriser l'innovation dans les entreprises, tels que des programmes d'articulation avec les laboratoires publics ou la réduction des frais d'enregistrement des nouveaux produits. D'autres actions concernent la formation des conseillers agricoles et, plus en amont, des ingénieurs agronomes dans les universités, sur les thèmes de la lutte biologique et de la biologie des sols. D'autres actions, plus modestes, encouragent l'adoption et l'usage de ces technologies par des subventions et par la sensibilisation auprès de groupements d'agriculteurs.

Mais ce sont également ces agriculteurs qui sont au cœur d'une autre dynamique, et sur laquelle le Brésil joue un rôle d'avant-garde : la production à la ferme d'intrants biologiques.

\section{Au Brésil, l'essor de la production des biointrants par les grandes exploitations agricoles}

La production d'intrants biologiques par les agriculteurs n'est pas une nouveauté — on parle de production à la ferme. Des agriculteurs et des organisations non gouvernementales promouvant les pratiques agroécologiques agissent dans ce sens depuis de nombreuses années.

La promotion et l'encadrement de la production à la ferme sont une des orientations du Programme national brésilien des biointrants (Programa Nacional de Bioinsumos). Dans certaines régions reculées, ou pour les petites exploitations familiales, l'accès aux intrants commerciaux reste en effet difficile et coûteux. De plus, la production et la manipulation de certains produits à base de microorganismes peuvent être sources de risques sanitaires. C'est pourquoi ce programme envisage de promouvoir l'installation de petites unités locales de production dans les territoires ruraux, les «biofabriques ». Celles-ci sont appelées à être gérées individuellement ou collectivement par des producteurs et à impliquer éventuellement des opérateurs privés ou coopératifs locaux.

Mais certains producteurs ont pris de court l'action publique et les industriels. Depuis le milieu des années 2010 s'est en effet structuré à l'échelle fédérale un réseau d'exploitations agricoles de grande taille, conventionnelles ou biologiques, pour encourager la production à la ferme et à grande échelle de microorganismes pour la fertilisation ou la santé des plantes. II s'agit du Groupe associé pour l'agriculture durable [GAAS, Grupo Asociado de Agricultura Sustentável], qui fédère des agriculteurs investissant dans des matériels vendus par des sociétés ayant pour clients habituels des laboratoires de recherche publique et des industries. Pour

\section{Le marché du biocontrôle en pleine expansion au Brésil}

Les données régionales concernant les marchés du biocontrôle et des biointrants font défaut. Pour le biocontrôle, voici quelques chiffres pour le Brésil, principal marché de la région, mis en regard de chiffres au niveau mondial.

Au Brésil, le marché total des produits chimiques et biologiques de protection des cultures est passé de 10,3 milliards à 11,6 milliards de dollars US entre les campagnes agricoles 2017-2018 et 2019-2020. La part des produits de biocontrôle y est minoritaire, mais elle est passée sur cette période de 1,5 à 2,6\%, c'est-à-dire un quasi doublement en valeur en trois ans. Au niveau mondial, les projections envisagent que le biocontrôle occupe 10 \% du marché des produits de protection des cultures en 2025 ; au Brésil, la proportion attendue est supérieure, autour de $14 \%$. Mais ces chiffres ne représentent que les ventes et n'incluent pas les intrants biologiques produits à la ferme - CropLife Brasil estime qu'environ $20 \%$ des surfaces traitées en biocontrôle au Brésil le sont avec ce type d'intrants.

Au niveau mondial, comme au Brésil, la croissance du marché du biocontrôle est de 15 \% par an. Au Brésil, le taux de croissance pronostiqué pour 2021 se situerait plutôt entre 20 et 30 \%. Entre 2020 et 2025, un doublement du marché brésilien est ainsi attendu, à l'image des perspectives qui se dessinent pour le marché mondial, dont le volume de vente en biocontrôle pourrait atteindre plus de 11 milliards de dollars US en 2025.

Au Brésil, les principales cultures sur lesquelles sont employés ces produits sont le soja, la canne à sucre, le café et le coton; à elles seules, elles représentent près des trois-quarts de la consommation nationale des produits de biocontrôle.

Cette progression brésilienne va de pair avec l'augmentation constante du nombre annuel d'enregistrements de nouveaux produits de biocontrôle, passant de 31 produits enregistrés en 2015 à 95 en 2020.

Ces chiffres illustrent la progression du marché et le dynamisme industriel autour du biocontrôle, au Brésil comme au niveau mondial. Mais la marge de progrès est encore immense, en particulier du côté de l'utilisation par les agriculteurs. En 2020, la plupart des agriculteurs brésiliens utilisant des produits de biocontrôle employaient en moyenne moins de deux produits par campagne agricole, le reste étant des traitements avec des intrants chimiques.

améliorer leurs pratiques, ces agriculteurs recrutent des ingénieurs microbiologistes et se font assister par des chercheurs de l'institut fédéral de recherche agricole, l'Embrapa (Empresa Brasileira de Pesquisa Agropecuária].

Cette dynamique pose certains problèmes à l'État et aux industries des biointrants en plein essor. L'État, avec le Programme national des biointrants, s'efforce de développer des outils de formation, de suivi et de contrôle pour encadrer sur le plan sanitaire ces pratiques innovantes. Les industries soutiennent quant à elles un encadrement le plus strict possible de ces pratiques de production à la ferme, de façon à garantir l'expansion d'un marché pour lequel elles investissent en recherche-développement. Elles veulent en effet garantir la propriété intellectuelle de leurs souches de microorganismes et font pression, sans avoir 
obtenu de mesure concrète à ce stade, pour interdire la reproduction à la ferme de microorganismes issus de leurs produits commerciaux, qu'elles considèrent comme de la «bio-piraterie ». Cette situation rappelle évidemment les mobilisations et les controverses qui ont éclaté autour des semences dans les années 1990. Les biointrants se situent ainsi au carrefour d'intérêts industriels et de mobilisations paysannes défendant des approches contrastées, voire antagonistes, de la gestion et de l'utilisation du vivant.
En France et en Europe, ces dynamiques de production à la ferme de microorganismes sont aujourd'hui absentes, ou quasiment inexistantes. Mais on y observe un intérêt grandissant pour le biocontrôle, pour l'agroécologie et pour la réappropriation par les agriculteurs de certaines activités dans la conception et l'usage des technologies. Il est de ce fait important de scruter avec attention les innovations à l'œuvre en Amérique du Sud et la façon dont elles contribuent à renouveler les relations entre agriculteurs, recherche agricole et industrie des intrants.
Ce Perspective $\mathrm{n}^{\circ} 55$ est issu de travaux de recherche conduits dans le cadre du réseau Politiques publiques et développement rural en Amérique latine et Caraïbe (dispositif de recherche et d'enseignement en partenariat (dP) du Cirad PP-AL, https://www.pp-al.org/].

Avec l'Institut interaméricain de coopération pour l'agriculture (IICA), le dP PP-AL a organisé entre juin et août 2020 une série de conférences dédiées à cinq expériences nationales de promotion des biointrants agricoles - Argentine, Brésil, Colombie, Équateur, Mexique. Les cinq conférences de ce cycle sont en ligne en accès libre à https://www.pp-al.org/actualites/politiques-publiques-etinnovation-en-faveur-des-bio-intrants-agricoles.

Certains de ces travaux se sont également déroulés dans le cadre du projet de recherche "Institutionnalisations des agroécologies " (IDAE, https://www.idae.cnrs.fr/, 2016-2019, Agence nationale de la recherche, Anr, France, projet 15-CE21-0006-01, https://anr.fr/Projet-ANR-15-CE21-0006). Les travaux au Brésil ont été menés avec l'aide de bourses de chercheurs visitants de la Fondation de soutien à la recherche de l'État de Rio de Janeiro (Fundação de Amparo à Pesquisa do Estado do Rio de Janeiro, FAPERJ, Brésil, PV 201.798/2017) et du Conseil national de développement scientifique et technologique (Conselho Nacional de Desenvolvimento Científico e Tecnológico, CNPq, Brésil, PV 301509/2018-2]
Ces travaux ont donné lieu aux publications suivantes:

Goulet F., 2021. Characterizing alignments in sociotechnical transitions. Lessons from agricultural bioinputs in Brazil. Technology in Society. https://doi.org/10.1016/j.techsoc.2021.101580

Goulet F., Hubert M., 2020. Making a Place for Alternative Technologies: The Case of Agricultural Bio-Inputs in Argentina. Review of Policy Research 37 [4]: 535-555. https://doi.org/10.1111/ropr.12384

Goulet F., Aulagnier A., Hubert M., 2020. Del reemplazo tecnológico al desplazamiento de fronteras: las alternativas a los agroquimicos en Argentina, Brasil y Francia. In: Mombello L., Spivak L'Hoste A. [Eds.), Naturaleza y conocimientos en tensión. Buenos Aires, TeseoPress, p. 279-299. ISBN 978-987-86-5724-0. https://www.teseopress.com/bienes/

Goulet F., Krotsch T., 15 septembre 2020. Políticas públicas para los bioinsumos: hacia un espacio de intercambio en América Latina y EI Caribe. IICA Blog. https://blog.iica.int/en/blog/politicas-publicaspara-los-bioinsumos-hacia-un-espacio-intercambio-en-americalatina-caribe

\section{Quelques liens}

CropLife Brasil, 2019. https://croplifebrasil.org/

Empresa Brasileira de Pesquisa Agropecuária (Embrapa). https://www.embrapa.br/

Inter-American Institute for Cooperation on Agriculture, IICA, 2020. https://www.iica.int/en

Ministère de l'agriculture et de l'alimentation (France), 2020. Stratégie nationale de déploiement du biocontrôle 2020-2025. Novembre 2020. 28 p. https://agriculture.gouv.fr/quest-ce-que-le-biocontrole Grupo Asociado de Agricultura Sustentável (GAAS, Brésil), 2020. http://www.grupoagrisustentavel.com.br

Ministério da Agricultura, Pecuária e Abastecimento (Brésil), 2021. Programa Nacional de Bioinsumos.

https://www.gov.br/agricultura/pt-br/assuntos/inovacao/bioinsumos Ministerio de Agricultura, Ganadería y Pesca (Argentine). Componentes de la Bioeconomía Bioinsumos / Biomateriales.

https://www.magyp.gob.ar/sitio/areas/observatorio_bioeconomia/ indicadores/04/index.php

\section{Quelques mots sur...}

Frédéric Goulet est sociologue au Cirad, Umr Innovation (Innovation et développement dans l'agriculture et l'alimentation https://umr-innovation.cirad.fr/). Depuis 2018, il est professeur visitant de l'Université fédérale rurale de Rio de Janeiro au Brésil (UFRRJ https://portal.ufrrj.br/) dans le cadre du Programme d'études supérieures de sciences sociales en développement, agriculture et société (Programa de Pós-Graduação de Ciências Sociais em Desenvolvimento, Agricultura e Sociedade, CPDA, https://institucional.ufrrj.br/portalcpda/). Il a été chercheur visitant (2013-2018] au sein de I'Instituto Nacional de Tecnología Agropecuaria (INTA, https://www.argentina.gob.ar/inta) en Argentine. frederic.goulet@cirad.fr

\section{Directeur de la publication : Michel Eddi, Président directeur général du Cirad Rédacteur.rice.s en chef : Patrick Caron, Umr ART-Dev [Acteurs, ressources et territoires dans le développement] Cécile Fovet-Rabot, délégation à l'information scientifique et technique Étienne Hainzelin, direction générale Mise en pages et illustrations : Laurence Laffont Diffusion : Christiane Mastri, délégation à la communication}

https://revues.cirad.fr/index.php/perspective perspactive ISSN-L 2275-9131 - Email : perspective@cirad.fr

Pour citer ce document

Goulet F., 2021. Intrants biologiques et politiques agricoles en Amérique du Sud : entre ruptures et continuités. Montpellier, Cirad, Perspective 55 https://doi.org/10.19182/perspective/36381 